OHSTPY-HEP-T-02-005

OSU-HEP-02-08

\title{
Magnetic Monopoles with Wilson loops on a 5D Orbifold
}

\author{
Radovan Dermíšek and Stuart Rabyர \\ Department of Physics, The Ohio State University, \\ $174 W$ 18th Ave, Columbus, OH 43210 \\ S. Nandif \\ Department of Physics, Oklahoma State University, Stillwater, OK 74078
}

(Dated: December 2, 2018)

\begin{abstract}
We discuss magnetic monopoles in gauge theories with Wilson loops on orbifolds. We present a simple example in 5 dimensions with the fifth dimension compactified on an $S^{1} / Z_{2}$ orbifold. The Wilson loop in this $S O(3)$ example replaces the adjoint Higgs scalar (needed to break $S O(3)$ to $U(1))$ in the well-known 't Hooft - Polyakov construction. Our solution is a magnetic monopole string with finite energy, and length equal to the size of the extra dimension.
\end{abstract}

*dermisek@pacific.mps.ohio-state.edu

traby@pacific.mps.ohio-state.edu

shaown@okstate.edu 


\section{INTRODUCTION}

Recently there has been great interest in non-abelian gauge field theories in $4+\mathrm{d}$ dimensions with d extra dimensions compactified on an orbifold [1, 2, 3, 田, 5]. The extra dimensions can have inverse radii of order a few $\mathrm{TeV}$, of order the GUT scale or anything in between. In most recent papers gauge and supersymmetry breaking, via boundary conditions imposed upon orbifold compactification, has been an interesting alternative to the traditional Higgs mechanism. As an illustrative and simple example, $S^{1} / Z_{2}$ and $S^{1} /\left(Z_{2} \times Z_{2}^{\prime}\right)$ in one extra dimension have been used to break the GUT groups $S U(5) \rightarrow S U(3) \times S U(2) \times U(1)$ [1], 2], $S O(10) \rightarrow S U(4) \times S U(2)_{L} \times S U(2)_{R}$ [3], the left-right gauge symmetry $S U(2)_{L} \times S U(2)_{R} \times U(1)_{B-L} \rightarrow S U(2)_{L} \times U(1)_{R} \times U(1)_{B-L}$ 《\# or the electroweak unified group $S U(3) \rightarrow S U(2) \times U(1)$ [5]. In this paper we argue that magnetic monopoles are generic consequences of gauge symmetry breaking with Wilson loops on $S^{1} / Z_{2}$ orbifolds. The proof is by construction. We show how to construct magnetic monopole (or more precisely, magnetic monopole string) solutions in these theories. We also elucidate the correspondence between compactification with Wilson loops on $S^{1} / Z_{2}$ and gauge symmetry breaking on $S^{1} /\left(Z_{2} \times Z_{2}^{\prime}\right)$ orbifolds.

Recall that Dirac [6] showed how to construct magnetic monopoles in $U(1)$ gauge theories. The Dirac monopole is singular at the origin and thus has infinite mass. In addition a magnetic flux tube (the so-called Dirac string) extends from the origin to spatial infinity. Dirac showed however that if and only if the monopole charge $g$ satisfies the quantization condition $g e=n / 2$, with $n \in \mathbb{Z}$ and $e$ the minimal electric charge, will the flux tube be an unobservable gauge artifact.

't Hooft and Polyakov [7] embedded the Dirac monopole into a non-abelian gauge theory, in particular Georgi-Glashow $S O(3)$ [8]. In this example $S O(3)$ is spontaneously broken to $U(1)_{E M}$ via the vacuum expectation value [vev] $(V)$ of a Higgs scalar in the adjoint representation. In the 't Hooft - Polyakov construction the monopole singularity is removed and the Dirac string is eliminated, resulting in a monopole with finite mass $\sim 4 \pi V / e$ and two units of Dirac magnetic charge with $g=1 / e$. Note, the 't Hooft - Polyakov monopole can be embedded into any $S U(N)$ gauge theory, see for example [9].

In gauge theories defined on $M \times \Gamma$ (with $M$ a four dimensional Minkowski space and $\Gamma$ a compact $d$ dimensional manifold with non-trivial homotopy $\Pi_{1}(\Gamma)$ ), Hosotani [10] showed 
that Wilson loops, i.e. $\exp \left(i \oint A_{\alpha} d x^{\alpha}\right)$ integrated around a non-contractible closed loop in $\Gamma$, can spontaneously break the gauge symmetry. The Wilson loop acts like the vev of a Higgs scalar in the adjoint representation. Wen and Witten [11] considered magnetic monopoles with Wilson loops on compact manifolds. They assumed $\Pi_{1}(\Gamma)=Z_{n}$ and discussed the allowed values of magnetic monopole charges in such theories. A simple explicit example of a monopole construction with Wilson loops was discussed by Lee et al. [12] using an $S U(3)$ gauge theory defined on $M \times S^{3} / Z_{2}$. In their example, the Wilson loop breaks $S U(3) \rightarrow S U(2) \times U(1)$.

In this paper, we extend the construction of magnetic monopoles to orbifolds [25]. In particular we consider the simple example of an $S O(3)$ gauge theory defined on the orbifold $M \times S^{1} / Z_{2}$ with a background gauge field, or equivalently, $M \times S^{1} /\left(Z_{2} \times Z_{2}^{\prime}\right)$. Our discussion is self-contained, however for a recent discussion of Wilson loops on orbifolds see [13, 14. Finally our analysis is easily extended to any $S U(N)$ gauge group defined on $M \times S^{1} / Z_{2}$ using previous analyses for the extension of 't Hooft - Polyakov monopoles [9].

The paper is organized as follows. In section II we introduce the Wilson loop symmetry breaking mechanism in the simple example of the circle $S^{1}$. We then generalize this discussion in section $\amalg I \mathrm{~A}$ to the orbifold $S^{1} / Z_{2}$. In ПIIB we elucidate the equivalence of gauge symmetry breaking with Wilson loops on $S^{1} / Z_{2}$ and gauge symmetry breaking on the orbifold $S^{1} /\left(Z_{2} \times Z_{2}^{\prime}\right)$. In section $\Pi 1 \mathrm{IT}$ we explicitly construct the monopole string solution and discuss some of its properties. Finally in $\mathbb{\square}$ we summarize our results and consider possible phenomenological ramifications.

\section{SO(3) GAUGE THEORY ON $M \times S^{1}$}

Consider a general gauge theory with symmetry group $G$ in five dimensional spacetime. The Lagrangian is given by

$$
\mathcal{L}_{5}=-\frac{1}{4 e_{5}^{2} k} \operatorname{Tr}\left(F_{M N} F^{M N}\right)
$$

where $F_{M N} \equiv \sum_{a} F_{M N}^{a} T^{a}, \quad T^{a}$ are generators in some finite dimensional representation of $G$ normalized such that $\operatorname{Tr}\left(T^{a} T^{b}\right)=k \delta^{a b}$ and $M, N=\{0,1,2,3,5\}$ :

$$
F_{M N}=\partial_{M} A_{N}-\partial_{N} A_{M}+i\left[A_{M}, A_{N}\right]
$$


(For the adjoint representation of $S O(3)$ we use the standard normalization of the generators with $k=2$.) The gauge transformation of the gauge field $A_{M}\left(x_{\mu}, y\right) \equiv \sum_{a} A_{M}^{a} T^{a}\left(x_{\mu}, y\right)$ (greek indices correspond to 4 -dimensional Minkowski spacetime and $y \equiv x_{5}$ ) is given by

$$
A_{M}\left(x_{\mu}, y\right) \rightarrow U A_{M}\left(x_{\mu}, y\right) U^{\dagger}-i U \partial_{M} U^{\dagger}
$$

where

$$
U=\exp \left(i \theta^{a}\left(x_{\mu}, y\right) T^{a}\right)
$$

In our notation, Eq. (1), the gauge fields have mass dimensions [1], and the charge $e_{5}$ has dimension [-1/2]. We can also define the effective four dimensional, dimensionless, gauge coupling $e$ by rescaling $e_{5}$ in Eq. (11) via the expression $e_{5}=\sqrt{2 \pi R} e$. Note, if $\partial_{5} A_{\mu}=0$, then $F_{\mu 5}$ reduces to the covariant derivative of the 5 th component of the gauge field $A_{5}$. In this case we can conveniently define $\Phi \equiv A_{5} / e_{5}=\tilde{\Phi} / \sqrt{2 \pi R}$, where the scalars $\Phi$ and $\tilde{\Phi}$ have dimension [3/2] and [1]. The Lagrangian (11) can then be rewritten in the suggestive form:

$$
\mathcal{L}_{5}=\frac{1}{2 \pi R}\left[-\frac{1}{4 e^{2} k} \operatorname{Tr}\left(F_{\mu \nu} F^{\mu \nu}\right)+\frac{1}{2 k} \operatorname{Tr}\left(D_{\mu} \tilde{\Phi} D^{\mu} \tilde{\Phi}\right)\right] .
$$

This resembles the Georgi-Glashow model [8] of an $S O(3)$ gauge theory interacting with an isovector Higgs field. There are two differences, however. First, there is no potential $V(\tilde{\Phi})=\lambda\left(\tilde{\Phi}^{a} \tilde{\Phi}^{a}-V^{2}\right)^{2}$ for the Higgs field which would break the gauge symmetry down to $U(1)$ and second, the Higgs field depends on the 5th coordinate. Although this analysis is limited to gauge fields satisfying $\partial_{5} A_{\mu}=0$, it nevertheless inspires the following discussion of symmetry breaking via Wilson loops and the further consideration of monopoles with Wilson loops. In general, however, $\partial_{5} A_{\mu} \neq 0$ and we need to keep the full $\operatorname{Tr}\left(F_{\mu 5}^{2}\right)$ term.

\section{A. Wilson loop gauge symmetry breaking on $M \times S^{1}$}

Assume the 5 th dimension is compactified on a circle $S^{1}$ parametrized by $y \in[0,2 \pi R]$. The gauge symmetry can then be broken by the presence of a background gauge field $A_{5}$. This symmetry breaking mechanism is known as Hosotani or Wilson loop symmetry breaking [10]. Consider the constant background to be along the third isospin direction,

$$
A_{5}(y)=A_{5}^{3} T^{3}
$$


Using the single valued gauge transformation (periodic under $y \rightarrow y+2 \pi R$ ) given by Eqs. (3,田) with $\theta\left(x_{\mu}, y\right)=-n y / R, n \in \mathbb{Z}$ :

$$
U(y)=\exp \left(-i n T^{3} \frac{y}{R}\right)
$$

we obtain the transformation of $A_{5}^{3}$ :

$$
A_{5}^{3} \rightarrow A_{5}^{3}+n / R
$$

Therefore the gauge non-equivalent values of $A_{5}^{3}$ can be chosen to lie between 0 and $1 / R$. The holonomy due to this constant background gauge field is given by

$$
T=\exp \left(i \oint A_{5} d y\right)=\exp \left(i \alpha T^{3}\right)
$$

with the arbitrary parameter $\alpha \equiv 2 \pi R A_{5}^{3}$. Note the set of possible holonomies $\left\{\mathbb{1}, T^{ \pm 1}, T^{ \pm 2}, \cdots\right\}$ provides a mapping of the gauge group into the discrete group $\mathbb{Z}$. This non-trivial holonomy affects the spectrum of the theory. A massless periodic scalar field $\phi$ (satisfying $\phi(y+2 \pi R)=\phi(y))$ with isospin eigenvalue $I_{3}$ can be decomposed into KaluzaKlein modes

$$
\phi_{(n)}\left(x_{\mu}\right) \exp (\text { iny } / R)
$$

The 5-dimensional wave equation $D^{M} D_{M} \phi=0$ splits into an infinite set of 4 -dimensional wave equations for Kaluza-Klein modes $\phi_{(n)}$ with masses given by

$$
m_{(n)}^{2} \phi_{(n)} \exp (i n y / R)=-\left(\partial_{y}+i A_{5}^{3} T^{3}\right)^{2} \phi_{(n)} \exp (i n y / R)=\left(\frac{n}{R}+A_{5}^{3} I_{3}\right)^{2} \phi_{(n)} \exp (i n y / R) .
$$

It is now easy to obtain the spectrum of gauge fields [26]. The gauge field $A_{\mu}^{3}(y)$ has $I_{3}=0$ and therefore its KK modes are not affected by the holonomy. The zero mode of this field corresponds to the gauge field of the unbroken $U(1)$. On the other hand, the masses of the KK modes of the $W^{ \pm}$gauge bosons, with $I_{3}= \pm 1$, are given by $m_{(n)}=\left|\frac{n}{R} \pm A_{5}^{3}\right|$. If $A_{5}^{3} \neq \frac{k}{R}$, where $k \in \mathbb{Z}$, the gauge bosons $W^{ \pm}$are all massive. Clearly the $S O(3)$ symmetry is broken to $U(1)$. Note, the symmetry breaking scale satisfies $0 \leq A_{5}^{3}<1 / R$, but is otherwise unconstrained. 


\section{B. Gauge Picture with Vanishing Background}

A constant background gauge field $A_{5}^{3}$ may be gauged away with the non-periodic gauge transformation

$$
U(y)=\exp \left(i y A_{5}^{3} T^{3}\right)
$$

In this gauge the covariant derivative in Eq. (11) is trivial, i.e. $D_{5}=\partial_{5}$. Nevertheless it is easy to see that, as expected, the physics is unchanged.

This gauge transformation is not single valued and thus the periodicity condition $\phi(y+2 \pi R)=\phi(y)$ becomes

$$
\phi(y+2 \pi R)=\exp \left(i \alpha T^{3}\right) \phi(y) .
$$

Now the mode expansions are of the form

$$
\phi_{(n)}\left(x_{\mu}\right) \exp \left[i\left(n / R+A_{5}^{3} I_{3}\right) y\right]
$$

resulting in the identical spectrum as before.

\section{SO(3) GAUGE THEORY ON $S^{1} / Z_{2}$}

\section{A. The $S^{1} / Z_{2}$ orbifold}

The $S^{1} / Z_{2}$ orbifold is a circle $S^{1}$ modded out by a $Z_{2}$ parity symmetry: $y \rightarrow-y$. The 5th dimension is now a line segment $y \in[0, \pi R]$. This orbifold has two fixed points at $y=0$ and $\pi R$. The Lagrangian (1) is invariant under the parity transformation

$$
\begin{gathered}
A_{\mu}(-y)=A_{\mu}(y) \\
A_{5}(-y)=-A_{5}(y) .
\end{gathered}
$$

As in the case of compactification on a circle we consider a constant background for $A_{5}^{3}$

(Eq. (6) ). Clearly such a background is not consistent with the parity operation, Eq. (16). However, following [13 we define a generalized parity by combining the parity transformation (16) with the gauge transformation (8), for $n=1, A_{5}^{3} \rightarrow A_{5}^{3}+1 / R$. We then look for a consistent solution with constant $A_{5}^{3}$. There are now only two possible values for $A_{5}^{3}$. The possibility $A_{5}^{3}=0$ is obviously allowed, but in this case the gauge symmetry is unbroken. 
The only nontrivial choice corresponds to $A_{5}^{3}(y)=\frac{1}{2 R}$ which changes sign under the "naive" parity, $A_{5}^{3}(-y)=-\frac{1}{2 R}$, but is gauge equivalent to its original value. Therefore, instead of (15) - (16) we define the fields for negative $y$, in the region $-\pi R<y<0$, in terms of the fields defined for positive $y$ in the fundamental domain, $0<y<\pi R$, via the generalized parity transformation (i.e. a combined "naive" parity transformation (16) and a gauge transformation) such that, in general:

$$
\begin{gathered}
A_{\mu}(-y)=U(-y) A_{\mu}(y) U^{\dagger}(-y)-i U(-y) \partial_{\mu} U^{\dagger}(-y), \\
A_{5}(-y)=-U(-y) A_{5}(y) U^{\dagger}(-y)-i U(-y) \partial_{-y} U^{\dagger}(-y),
\end{gathered}
$$

with

$$
U(y)=\exp \left(-i \frac{y}{R} T^{3}\right)
$$

It is useful to define new fields, $W^{ \pm}$, in a usual way from $A^{1}$ and $A^{2}$ :

$$
W^{ \pm}=\frac{1}{\sqrt{2}}\left(A^{1} \mp i A^{2}\right), \quad T^{ \pm}=\frac{1}{\sqrt{2}}\left(T^{1} \pm i T^{2}\right) .
$$

With this definition we have $A^{1} T^{1}+A^{2} T^{2}=W^{+} T^{+}+W^{-} T^{-}$and $\left[T^{3}, T^{ \pm}\right]= \pm T^{ \pm}$. Using the identity

$$
\exp \left(i \frac{y}{R} T^{3}\right) T^{ \pm} \exp \left(-i \frac{y}{R} T^{3}\right)=\exp \left( \pm i \frac{y}{R}\right) T^{ \pm}
$$

it is easy to show that the generalized parity tranformation acts on gauge fields as follows:

$$
\begin{aligned}
W_{\mu}^{ \pm}(-y) & =\exp \left( \pm i \frac{y}{R}\right) W_{\mu}^{ \pm}(y) \\
W_{5}^{ \pm}(-y) & =-\exp \left( \pm i \frac{y}{R}\right) W_{5}^{ \pm}(y), \\
A_{\mu}^{3}(-y) & =A_{\mu}^{3}(y) \\
A_{5}^{3}(-y) & =-A_{5}^{3}(y)+\frac{1}{R} .
\end{aligned}
$$

To summarize, using a more compact notation, we have the following constraints on the fields (valid for all modes, except the constant piece of $A_{5}^{3}$ ). Under the generalized parity transformation the fields $\phi_{P}$ (with $P= \pm 1$ ) satisfy:

$$
\phi_{P}(-y)=P \exp \left(i \frac{y}{R} I_{3}\right) \phi_{P}(y)
$$

with isospin eigenvalue $I_{3}= \pm 1,0$. The periodicity condition is given by:

$$
\phi_{P}(y+2 \pi R)=\phi_{P}(y)
$$


We then obtain the following decomposition into KK modes:

$$
\begin{array}{r}
\phi_{+}\left(x_{\mu}, y\right)=\sum_{n=0}^{\infty} \phi_{+}^{(n)}\left(x_{\mu}\right) \exp \left(-i \frac{y}{2 R} I_{3}\right) \cos n \frac{y}{R} \quad \text { for even } I_{3}, \\
\phi_{+}\left(x_{\mu}, y\right)=\sum_{n=0}^{\infty} \phi_{+}^{(n)}\left(x_{\mu}\right) \exp \left(-i \frac{y}{2 R} I_{3}\right) \cos (n+1 / 2) \frac{y}{R} \quad \text { for odd } I_{3}, \\
\phi_{-}\left(x_{\mu}, y\right)=\sum_{n=0}^{\infty} \phi_{+}^{(n)}\left(x_{\mu}\right) \exp \left(-i \frac{y}{2 R} I_{3}\right) \sin (n+1) \frac{y}{R} \quad \text { for even } I_{3}, \\
\phi_{-}\left(x_{\mu}, y\right)=\sum_{n=0}^{\infty} \phi_{+}^{(n)}\left(x_{\mu}\right) \exp \left(-i \frac{y}{2 R} I_{3}\right) \sin (n+1 / 2) \frac{y}{R} \quad \text { for odd } I_{3} .
\end{array}
$$

From transformations (22) - (25) we see that the KK mode expansion of $A_{\mu}^{3}[(+)$ field with $I_{3}=0$ ] is given in Eq. (28) with corresponding masses $n / R$. This is the only field which has a zero mode. It corresponds to the gauge field of the unbroken $U(1)$. The expansion of $W_{\mu}^{ \pm}$ $\left[(+)\right.$ field with $\left.I_{3}= \pm 1\right]$ is given in Eq. (29) with corresponding masses $(n+1 / 2) / R$. Similarly, the expansion of $W_{5}^{ \pm}\left[(-)\right.$field with $\left.I_{3}= \pm 1\right]$ is given in Eq. (31) with corresponding masses $(n+1 / 2) / R$. And finally, the expansion of $A_{5}^{3}\left[(-)\right.$ field with $\left.I_{3}=0\right]$ is given by Eq. (30) up to the value of the constant background:

$$
A_{5}^{3}\left(x_{\mu}, y\right)=\frac{1}{2 R}+\sum_{n=0}^{\infty} A_{5}^{3(n)}\left(x_{\mu}\right) \sin (n+1) \frac{y}{R} .
$$

The holonomy $T$ in this case is given by

$$
T=\exp \left(i \oint A_{5}^{3} T^{3}\right)=\exp \left(i \pi T^{3}\right)=\operatorname{diag}(-1,-1,1) .
$$

Hence $T^{2}=\mathbb{1}$ or the set of possible holonomies $\{\mathbb{1}, T\}$ maps the gauge group into the discrete group $\mathbb{Z}_{2}$. Unlike the case of Wilson loops on $S^{1}$ discussed in section II, the background gauge field and consequently the holonomy on $S^{1} / Z_{2}$ can only take discrete values.

Now let us consider the gauge picture with vanishing background gauge field. As in the case of compactification on a circle, we can gauge away the constant background by the non-single valued gauge transformation given in Eq. (12). The transformations under the generalized parity are now those of Eqs. (15) and (16). In addition the non-single valued gauge transformation changes the periodicity condition as in Eq. (13) with $\alpha=\pi$.

To obtain the spectrum of KK modes of a field $\phi$ we consider both the transformation under parity and the effect of a non-trivial holonomy. Under parity,

$$
\mathcal{P}: \quad \phi_{P T}(y) \rightarrow \phi_{P T}(-y)=P \phi_{P T}(y),
$$


with $P^{2}=1$ or $P= \pm 1$. When going around the circle, the fields transform in the following way:

$$
\mathcal{T}: \quad \phi_{P T}(y) \rightarrow \phi_{P T}(y+2 \pi R)=T \phi_{P T}(y)
$$

with $T^{2}=\mathbb{1}$ or $T= \pm 1$. Therefore there are four different kinds of fields $\phi_{ \pm \pm}$corresponding to the four different combinations of $(P, T)$. It is easy to see that a field with given $(P, T)$ can be expanded into the following modes:

$$
\begin{aligned}
& \xi_{n}(+,+)=\cos n \frac{y}{R} \\
& \xi_{n}(+,-)=\cos (n+1 / 2) \frac{y}{R} \\
& \xi_{n}(-,+)=\sin (n+1) \frac{y}{R} \\
& \xi_{n}(-,-)=\sin (n+1 / 2) \frac{y}{R}
\end{aligned}
$$

Only the $(+,+)$ fields have massless zero modes. Of all the gauge fields only $A_{\mu}^{3}$ is a $(+,+)$ field with a zero mode. $W_{\mu}^{ \pm}, A_{5}^{3}$ and $W_{5}^{ \pm}$are $(+,-),(-,+)$and $(-,-)$fields, respectively. Clearly the mode expansion and the corresponding KK masses are the same as in the previous picture. Note, our gauge transformation parameters (Eq. (田) are constrained to satisfy $\theta^{3}\left(x_{\mu}, y\right)=\theta_{n}^{3}\left(x_{\mu}\right) \xi_{n}(+,+)$ and $\theta^{1,2}\left(x_{\mu}, y\right)=\theta_{n}^{1,2}\left(x_{\mu}\right) \xi_{n}(+,-)$. Hence, $S O(3)$ is the symmetry everywhere in the five dimensions, EXCEPT on the boundary at $y=\pi R$.

\section{B. Correspondence to $S^{1} /\left(Z_{2} \times Z_{2}^{\prime}\right)$ orbifold}

The $S^{1} / Z_{2}$ orbifold with holonomy $T$ in the gauge picture without a constant background gauge field is directly related to the $S^{1} /\left(Z_{2} \times Z_{2}^{\prime}\right)$ orbifold used recently in the literature [1, 2, 3, 目, 岛. This correspondence is also evident in the work of Ref. [13, 15]. We just need to identify the $S^{1} /\left(Z_{2} \times Z_{2}^{\prime}\right)$ orbifold with $S^{1}$, a circle of circumference $4 \pi R$, divided by the $Z_{2}$ transformation $y \rightarrow-y$ and $Z_{2}^{\prime}$ transformation $y^{\prime} \rightarrow-y^{\prime}$, where $y^{\prime} \equiv y-\pi R$. The

physical space is again the line segment $y \in[0, \pi R]$ with orbifold fixed points at $y=0$ and $\pi R$. It is easy to see that $\mathcal{P}^{\prime} \in Z_{2}^{\prime}$ in this picture corresponds to the combined translation and parity transformation in the previous picture, namely $\mathcal{P}^{\prime}=\mathcal{T} \mathcal{P}$. Note, a point at $y=y_{0}$ which corresponds to $y^{\prime}=y_{0}-\pi R$ is transformed by $Z_{2}^{\prime}$ into the point $y^{\prime}=-\left(y_{0}-\pi R\right)$ corresponding to $y=-y_{0}+2 \pi R$; this is equivalent to the action of $\mathcal{T} Z_{2}$ on the point at $y=y_{0}$, see Fig. [1. 

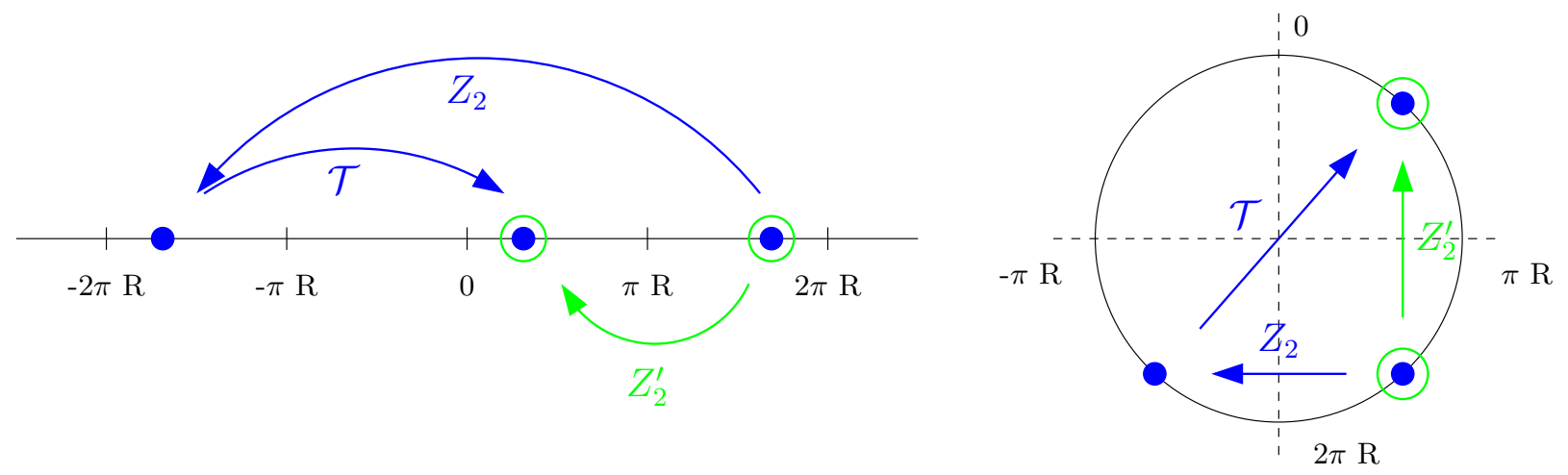

FIG. 1: The $Z_{2}^{\prime}$ parity transformation is equivalent to the combined $Z_{2}$ parity transformation and translation $\mathcal{T}$.

The action of $Z_{2}$ on the fields is given by

$$
\mathcal{P}: \quad \phi_{P P^{\prime}}(y) \rightarrow \phi_{P P^{\prime}}(-y)=P \phi_{P P^{\prime}}(y)
$$

with $P^{2}=1$ or $P= \pm 1$. Similarly, under $Z_{2}^{\prime}$ we have

$$
\mathcal{P}^{\prime}: \quad \phi_{P P^{\prime}}\left(\pi R+y^{\prime}\right) \rightarrow \phi_{P P^{\prime}}\left(\pi R-y^{\prime}\right)=P^{\prime} \phi_{P P^{\prime}}\left(\pi R+y^{\prime}\right)
$$

with $P^{\prime}=T P$ and $\left(P^{\prime}\right)^{2}=\mathbb{1}$ or $P^{\prime}= \pm 1$.

It is easy to see what the holonomy means in this picture. Since points $y_{0}$ and $y_{0}+2 \pi R$ are identified, the closed loop corresponds to going around half of the circle (the circumference of the circle in this picture is $4 \pi R$ ). Going around the whole circle (from $y_{0}$ to $y_{0}+2 \pi R$ and then from $y_{0}+2 \pi R$ to $y_{0}+4 \pi R$ ) clearly corresponds to $T^{2}$. From Eq. (16) we see that going from $y_{0}+2 \pi R$ to $y_{0}+4 \pi R$ is equivalent to going backwards from $y_{0}+2 \pi R$ to $y_{0}$. Therefore $T^{2}=\mathbb{1}$ and there are only two possibilities for holonomy, $T=+1$ and $T=-1$, the same as in the $S^{1} / Z_{2}$ picture. Hence we have $T \in \mathbb{Z}_{2}$. Note, in the above we have assumed that $P$ and $T$ can be simultaneously diagonalized. In general however $P$ and $T$ do not commute. In this case we would have $P T P=T^{-1}$.

\section{Monopole string on $S^{1} / Z_{2}$}

We saw that the gauge theory in 5-dimensions becomes a "gauge - Higgs" theory after the 5th dimension is compactified. The Higgs potential which breaks the $S O(3)$ gauge symmetry to $U(1)$ is absent, however its effect can be replaced by the Wilson loop along the 
compactified dimension. It was shown by 't Hooft and Polyakov [7] that the Georgi-Glashow model has a magnetic monopole solution to the equations of motion. It is natural to ask whether magnetic monopoles are present in the compactified 5-dimensional gauge theory and what is the correspondence with the usual 't Hooft - Polyakov solution.

The equations of motion corresponding to the Lagrangian (5) are:

$$
D_{\mu} D^{\mu} \tilde{\Phi}=0, \quad D_{\nu} F^{\mu \nu}=i e^{2}\left[\tilde{\Phi}, D^{\mu} \tilde{\Phi}\right]
$$

They correspond to the equations of motion of the Georgi-Glashow model in the absence of the Higgs potential.

Consider the ansatz (for $0<y<\pi R$ ):

$$
\begin{gathered}
A_{5} / e \equiv \tilde{\Phi}=\frac{1}{2 R e}(\hat{\vec{r}} \cdot \vec{T}) F(r), \\
A_{i}=-\frac{1}{r}(\vec{T} \times \hat{\vec{r}})_{i} G(r), \quad A_{0}=0,
\end{gathered}
$$

where $r=\sqrt{x_{i}^{2}}, \hat{r}_{i}=x_{i} / r$ and $F(r)$ and $G(r)$ are dimensionless functions. Asymptotically, for $r \rightarrow \infty$ we have $G(r) \rightarrow 1$. Note, the constant $\frac{1}{2 R}$ in the normalization of $A_{5}$ has been fixed by the vacuum boundary conditions with the choice $F(r) \rightarrow 1$ as $r \rightarrow \infty$ (see discussion below). This is exactly the 't Hooft-Polyakov ansatz, and therefore it is a solution to the equations of motion, Eq. (39) with

$$
V \equiv \lim _{r \rightarrow \infty} \sqrt{\operatorname{Tr}\left(\tilde{\Phi}^{2}\right) / k}=\frac{1}{2 R e} .
$$

In order to complete the solution we need to extend the above solution to negative $y$ (i.e. $-\pi R<y<0)$. As in the case with a constant background field $A_{5}$ we use the generalized parity operation, Eqs. (17) and (18), now with

$$
U=\exp \left(-i \frac{y}{R} \hat{\vec{r}} \cdot \vec{T}\right)
$$

we obtain

$$
\begin{gathered}
A_{5}(-y) / e \equiv \tilde{\Phi}(-y)=\frac{-F(r)+2}{2 R e}(\hat{\vec{r}} \cdot \vec{T}) \\
A_{i}(-y)=-\frac{G(r)-1}{r}(\vec{T} \times \hat{\vec{r}})_{i} \cos \frac{y}{R}+\frac{G(r)-1}{r}\left(T_{i}-\hat{r}_{i}(\hat{\vec{r}} \cdot \vec{T})\right) \sin \frac{y}{R}-\frac{1}{r}(\vec{T} \times \hat{\vec{r}})_{i} .
\end{gathered}
$$

Note, that the asymptotic values of $A_{i}$ and $A_{5}$, normalized as in Eq. (40), for $r \rightarrow \infty$ satisfy $A_{i}(-y)=A_{i}(y)$ and $A_{5}(-y)=A_{5}(y)$. Hence we obtain the asymptotic holonomy

$$
\lim _{r \rightarrow \infty} T(r)=\exp (i \pi \hat{\vec{r}} \cdot \vec{T})
$$


satisfying the condition $T^{2}=\mathbb{1}$, i.e. $T \in \mathbb{Z}_{2}$. Moreover in any given spatial direction $\hat{\vec{r}}$, the asymptotic holonomy is gauge equivalent to the vacuum value, Eq. (33). It is this physical requirement, that asymptotically far away from the monopole we recover the vacuum holonomy, which fixes the asymptotic magnitude of $A_{5}$, Eq. (40). Note, in the case of a simple circle, discussed in section $\mathbb{\Pi A}, T \in \mathbb{Z}$ and the magnitude of $A_{5}$ is arbitrary. In this case, the monopole mass can be taken continuously to zero. Hence monopoles on $S^{1}$ are unstable.

Although the form of the gauge fields for $-\pi R<y<0$, defined by the generalized parity transformation of the 't Hooft ansatz for $0<y<\pi R$ is quite complicated, it is easy to see that they are a solution to the field equations. This is because the action is both parity and gauge invariant. In fact the action

$$
S \equiv \int d^{4} x \int_{-\pi R}^{+\pi R} d y \mathcal{L}=2 \int d^{4} x \int_{0}^{+\pi R} d y \mathcal{L}
$$

is completely defined in terms of the fields in the fundamental domain $0 \leq y \leq \pi R$.

The asymptotic $(r \rightarrow \infty)$ gauge field strength is given by

$$
F_{i j}=-\frac{\epsilon_{i j k} \hat{r}_{k}(\hat{\vec{r}} \cdot \vec{T})}{r^{2}} .
$$

The asymptotic $U(1)$ abelian magnetic field is then given by

$$
B_{i} \equiv-\frac{1}{2 e k} \epsilon_{i j k} \operatorname{Tr}\left((\hat{\tilde{\Phi}}) F_{j k}\right)=\frac{\hat{r}_{i}}{e r^{2}}
$$

where $\hat{\tilde{\Phi}} \equiv \tilde{\Phi} / V$. Therefore the solution is a magnetic monopole string with total magnetic charge $g=1 / e$ or equivalently a magnetic charge per unit length in the 5 th direction given by $g / \pi R$.

The monopole string energy density is given by

$$
\mathcal{H}=\frac{1}{2 \pi R}\left[\frac{1}{4 e^{2} k} \operatorname{Tr}\left(F_{i j} F^{i j}\right)+\frac{1}{2 k} \operatorname{Tr}\left(D_{i} \tilde{\Phi} D_{i} \tilde{\Phi}\right)\right] .
$$

It is a constant function of $y$ and thus we should really talk about a monopole string stretched in the 5th direction from $y=0$ to $y=\pi R$. The energy density, Eq. (50), is the usual four dimensional energy density divided by the length of the fifth dimension and the energy per unit length of the monopole string is obtained by integrating $\mathcal{H}$ over the three flat spatial dimensions. Note, the integrated energy density from Eq. (50) can be written as

$$
H=\int d^{3} x \frac{1}{k} \operatorname{Tr}\left[\frac{1}{4}\left(\frac{1}{e} F_{i j} \mp \epsilon_{i j k} D_{k} \tilde{\Phi}\right)^{2} \pm \frac{1}{2 e} \epsilon_{i j k} F_{i j} D_{k} \tilde{\Phi}\right] .
$$


where the integration over $y$ has been performed. The second term can be rewritten using Bianchi identity as $\frac{1}{2 e k} \epsilon_{i j k} \partial_{k} \operatorname{Tr}\left(F_{i j} \tilde{\Phi}\right)$ and its contribution to the energy of the monopole is

$$
\pm \frac{1}{2 e k} \epsilon_{i j k} \int d^{3} x \partial_{k} \operatorname{Tr}\left(F_{i j} \tilde{\Phi}\right)= \pm V \int \vec{B} \cdot d \vec{S}= \pm 4 \pi V g
$$

When the first term in (51) vanishes the monopole solution is said to satisfy the Bogomol'nyi bound and such monopoles are called BPS monopoles. In fact, the general 't Hooft Polyakov monopole solution reduces to a BPS monopole in the limit that the Higgs potential for the adjoint scalar vanishes. Hence our monopole strings are in fact BPS monopole strings and their mass is given by

$$
M_{m}=\frac{4 \pi V}{e}=\frac{M_{W}}{\alpha}=\frac{1}{2 \alpha R}
$$

where $\alpha=e^{2} / 4 \pi$ is the dimensionless fine structure constant at the scale $1 / R$, and $R$ is the orbifold radius.

It is also important to express the equations for the BPS condition and the monopole energy density in an explicitly gauge invariant and 5D covariant form. The BPS condition is

$$
F_{i j}= \pm \epsilon_{i j k} F_{k 5}
$$

and the energy density is given by

$$
\mathcal{H}= \pm \frac{1}{2 e_{5} k} \epsilon_{i j k} \operatorname{Tr}\left(F_{i j} D_{k} \Phi\right)= \pm \frac{1}{2 e_{5}^{2} k} \epsilon_{i j k} \operatorname{Tr}\left(F_{i j} F_{k 5}\right)= \pm \frac{1}{8 e_{5}^{2} k} \epsilon_{0 N P Q R} \operatorname{Tr}\left(F^{N P} F^{Q R}\right) \text {. }
$$

Note it is then clear that the five dimensional Hamiltonian density is the time component of a five vector given by

$$
\mathcal{P}^{M}= \pm \frac{1}{8 e_{5}^{2} k} \epsilon^{M N P Q R} \operatorname{Tr}\left(F_{N P} F_{Q R}\right) \equiv \partial_{N} K^{M N}
$$

with

$$
K^{M N}= \pm \frac{1}{4 e_{5}^{2} k} \epsilon^{M N P Q R} \operatorname{Tr}\left(A_{P} F_{Q R}-i \frac{2}{3} A_{P} A_{Q} A_{R}\right)
$$

Hence $P^{M}$ satisfies the topological conservation law $\partial_{M} P^{M} \equiv 0$.

As a final note we can also consider the monopole solution in the gauge with vanishing background gauge field, i.e. $\left\langle A_{5}\right\rangle \equiv 0$. We find (for $0<y<\pi R$ )

$$
A_{5} / e \equiv \tilde{\Phi}=\frac{F(r)-1}{2 R e}(\hat{\vec{r}} \cdot \vec{T})
$$




$$
A_{i}=-\frac{G(r)-1}{r}(\vec{T} \times \hat{\vec{r}})_{i} \cos \frac{y}{2 R}+\frac{G(r)-1}{r}\left(T_{i}-\hat{r}_{i}(\hat{\vec{r}} \cdot \vec{T})\right) \sin \frac{y}{2 R}-\frac{1}{r}(\vec{T} \times \hat{\vec{r}})_{i} .
$$

Then for $-\pi R<y<0$ we obtain, by explicitly gauge transforming the fields in Eqs. (44) and (45), $A_{5}(-y)=-A_{5}(y)$ and $A_{i}(-y)=A_{i}(y)$ as expected from "naive" parity, Eqs. (15) and (16).

\section{CONCLUSIONS}

In this paper we have discussed Wilson loop symmetry breaking on orbifolds in five dimensions. We have tried to make the discussion of symmetry breaking on orbifolds self contained. We have also cleared up, in our minds, the mathematical correspondence between $S^{1} / Z_{2}$ orbifolds with a background gauge field and $S^{1} /\left(Z_{2} \times Z_{2}^{\prime}\right)$ orbifolds which have been considered in the literature. In fact they are identical upon rescaling the radius by a factor of 2. Although our analysis has been in non-supersymmetric gauge theories, it should be easy to extend to the case of orbifold symmetry breaking in supersymmetric gauge theories.

We have constructed monopole string solutions for an $S O(3)$ gauge group; valid when $S O(3)$ is broken to $U(1)$. Our construction can be extended to any $S U(N)$ gauge group on an $M \times S^{1} / Z_{2}$ orbifold with background gauge field. Such monopole strings may have interesting phenomenological consequences for grand unified scenarios with large extra dimensions [1]. They would be expected to have mass of order $1 / 2 \alpha R$, with a compactification scale $1 / R$ as small as a few TeV. Note that a GUT monopole string can lead to catalysis of baryon number violating processes [16].

Another interesting example would be in the case of the $S U(3)$ electroweak unification model recently discussed in the literature [5]. It is easy to show that this model also contains monopole strings when the symmetry is broken to either $S U(2) \times U(1)_{Y}$ or directly to $U(1)_{E M}$ with the addition of a Higgs multiplet in the triplet representation. Such a monopole string will have mass of order $60 / R$.

Clearly in light of the results presented here, it will be interesting to study monopole string production at high energy accelerators and at finite temperatures in the early universe. Tree level monopole string pair production cross sections at high energy colliders can be obtained from the usual Drell-Yan formulae, renormalized by the coupling of the monopole string, $e \rightarrow 1 / e$, ignoring any form factor effects [27]. If the monopoles are light enough, they can 
be pair produced in existing colliders such as the Fermilab Tevatron, and trapped and bound in the matter surrounding the collision region. Current bounds, using the samples exposed in the D0 experiment, on the production cross section of such monopole pairs is $0.42 \mathrm{pb}$, yielding a monopole mass limit of about $355 \mathrm{GeV}$ [17]. LHC should be able to push the limits to several $\mathrm{TeV}$, or observe signals.

The mass of the monopole string, discussed in this paper, depends on the orbifold compactification scale. Current collider bounds on the compactification scale depend on the scenario for the Standard Model particles. If only gauge fields propagate in the extra dimension, $y$, then the bound on $1 / R$ is about a $\mathrm{TeV}$; hence the mass of the monopole string can be about $60 \mathrm{TeV}$ or higher. If all the SM particles propagate in the extra dimensions (the case of so-called Universal Extra Dimensions), the collider bound is significantly lower, around $350 \mathrm{GeV}$ 18. In this case, the monopole string could be as light as about $20 \mathrm{TeV}$. Such monopole pairs will be copiously produced in future very high energy hadron colliders, such as the proposed VLHC, via Drell-Yan pair production.

Finally we expect that at temperatures much above the compactification scale, the thermal averaged holonomy will vanish and the monopole solutions will cease to be relevant. Then as the universe cools below the compactification scale, the universe is expected to go through a symmetry breaking phase transition in which monopoles will be produced via the Kibble mechanism with roughly one monopole per horizon size at the transition temperature [19]. Of course any constraints from bounds on cosmological monopole fluxes will require detailed finite temperature analyses. There are very stringent limits on monopole fluxes, both laboratory and galactic. The galactic limit on the monopole flux $(F)$ is $F<10^{-15} / \mathrm{cm}^{2} / \mathrm{sec} / \mathrm{str}$, the so called "Parker Limit" [20]. A monopole with mass less than about $10^{7} \mathrm{TeV}$ will satisfy the Parker Limit. Also, such a relativistic monopole will not over close the universe. It has been suggested [21] that such monopoles may be the cosmic ray primary responsible for producing the ultra high energy cosmic rays 22] observed on earth going beyond the GZK cut off [23]. The mass of the monopole string solutions, presented in this paper, is determined by the compactification scale which could be around $10^{7}$ $\mathrm{TeV}$. Thus these monopole strings could be a candidate for the above scenario. The laboratory bound for the flux of monopoles of any mass and with any velocity passing through the Earth's surface is $7.2 \times 10^{-13} / \mathrm{cm}^{2} / \mathrm{sec} / \mathrm{str}$ [24]. 


\section{Acknowledgments}

S.R. and R.D. would like to thank S. Mathur and C. Imbimbo for discussions. R.D.

and S.R. received partial support for this work from DOE contract DOE/ER/01545-831. The work of SN was supported in part by US DOE Grants DE-FG030-98ER-41076 and DE-FG-02-01ER-45684.

[1] K.R. Dienes, E. Dudas, and T. Gherghetta, Phys. Lett. B436, 55 (1998), Nucl. Phys. B537, 47 (1999); N. Weiner, hep-ph/0106097.

[2] Y. Kawamura, Prog. Theor. Phys. 103, 613 (2000), Prog. Theor. Phys. 105, 691 (2001); L.J. Hall and Y. Nomura, Phys. Rev. D64, 055003 (2001); A. Hebecker and J. March-Russell, Nucl. Phys. B613, 3 (2001), Nucl. Phys. B625, 128 (2002).

[3] R. Dermíšek and A. Mafi, Phys. Rev. D65, 055002 (2002).

[4] Y. Mimura and S. Nandi, hep-ph/0203126.

[5] S. Dimopoulos, D.E. Kaplan and N. Weiner, hep-ph/0202136; S. Dimopoulos and D.E. Kaplan, Phys. Lett. B531, 127 (2002); T. Li and L. Wei, hep-ph/0202090; L.J. Hall and Y. Nomura, hep-ph/0202107.

[6] P.A.M. Dirac, Proc. Roy. Soc. A133, 60 (1934); Phys. Rev. 74, 817 (1948).

[7] G. 't Hooft, Nucl. Phys. B79 276 (1974); A. M. Polyakov, JETP Lett. 20194 (1974).

[8] H. Georgi and S. L. Glashow, Phys. Rev. D6, 2977 (1972).

[9] A.S. Goldhaber and D. Wilkinson, Nucl. Phys. B114, 317 (1976); D. Wilkinson and A.S. Goldhaber, Phys. Rev. D16, 1221 (1977).

[10] Y. Hosotani, Phys. Lett. B126, 309 (1983); ibid Phys. Lett. B129, 193 (1983);

P. Candelas, G. Horowitz, A. Strominger and E. Witten, Nucl. Phys. B258, 46 (1985);

E. Witten, Nucl. Phys. B258, 75 (1985).

[11] X.-G. Wen and E. Witten, Nucl. Phys. B261, 651 (1985).

[12] B.-H. Lee, S.H. Lee, E.J. Weinberg and K. Lee, Phys. Rev. Lett. 60, 2231 (1988).

[13] L.J. Hall, H. Murayama and Y. Nomura, hep-th/0107245 (2001).

[14] M. Kubo, C.S. Lim, H. Yamashita, hep-ph/0111327.

[15] R. Barbieri, L.J. Hall, and Y. Nomura, Nucl. Phys. B624, 63 (2002). 
[16] V. Rubakov, JETP Lett. 33, 644 (1981), Nucl. Phys. B203, 311 (1982); C.G. Callan, Jr., Phys. Rev. 25, 2141 (1982).

[17] G. R. Kalbfleisch et al., Phys. Rev. Lett. 85, 5292 (2000); See also B. Abott etal, D0 Collaboration, Phys. Rev. Lett. 81, 524 (1998); M. Bertini etal., Eur. Phys. Lett. 12, 613 (1990).

[18] T. Appelquist, H-C. Cheng and B. A. Dobrescu, Phys. Rev. D64, 035002 (2001); T.G. Rizzo, Phys. Rev. D64, 095010 (2001); C. Macesanu, C.D. McMullen and S. Nandi,hep-ph/0201300; T. Appelquist and B. A. Dobrescu, Phys. Lett. B516, 85 (2001); K. Agashe, N.G. Deshpande, Phys. Lett. B514, 309 (2001); F. J. Petriello, hep-ph/0204067.

[19] T.W.B. Kibble, J. Phys. A 9, 1387 (1976); T.W.B. Kibble and Erick J. Weinberg, Phys. Rev. D43, 3188 (1991).

[20] E.N. Parker, Astrophys. J. 160, 383 (1970), Astrophys. J. 163, 225 (1971), Astrophys. J. 166, 295 (1971); M.S. Turner, E.N.Parker, and T. Bogdan, Phys. Rev. D26, 1296 (1982).

[21] T.W. Kephart and T. J. Weiler, Astropart. Phys. 4, 271 (1996); S.D. Wick, T.W. Kephart and T.J Weiler, hep-ph/0102002; T.J. Weiler, hep-ph/0103023, and references therein.

[22] See recent reviews by, P. Bierman, J. Phys. G23, 1 (1997); P. Bhattacharjee and G. Sigl, Phys. Rep. 327, 109 (2000); A.V. Olinto, Phys. Rep. 333, 329 (2000).

[23] K. Greisen, Phys. Rev. Lett. 16, 748 (1966); G.T. Zatsepin and V.A. Kuzmin, Pisma Zh Eksp. Teor Fiz 4, 114 (1966).

[24] M. E. Huber, B. Cabrera, M.A. Taber, and R.D. Gardner, Phys. Rev. Lett. 64, 835 (1990), Phys. Rev. D44, 636 (1991).

[25] These are actually monopole strings since they have constant energy density and charge in the fifth direction.

[26] Consider a background field gauge with $A_{M}=B_{M}+a_{M}$ where $B_{M}$ is the background value of the gauge field and $a_{M}$ are the small fluctuations. The background covariant derivative is given by $D_{M} \equiv \partial_{M}+i\left[B_{M},\right]$. If we use the covariant gauge fixing condition $D^{M} a_{M} \equiv 0$, then the gauge field equations of motion are given by $D^{M} D_{M} a_{N}+3 i\left[B_{N M}, a^{M}\right]=0$. Note, for a constant background gauge field $B_{N M} \equiv 0$.

[27] Tree level cross sections can be suitably "unitarized" to satisfy unitarity. 